Research Paper

\title{
Physical Exercise and Quality of Liie in Breast Cancer Survivors
}

\section{Marco VALENTI ${ }^{1}$, Giampiero PORZIO ${ }^{2}$, Federica AIELLI ${ }^{2}$, Lucilla VERNA ${ }^{2}$, Katia CANNITA ${ }^{2}$, Renato MANNO $^{3}$, Francesco MASEDU1 1 , Paolo MARCHETTI ${ }^{4}$, Corrado FICORELLA ${ }^{4}$}

1. Biostatistics and Epidemiology Unit, Faculty of Movement and Sport Science, and Department of Medicine and Public Health, University of L'Aquila. L'Aquila, Italy

2. Supportive Care and Rehabilitation Unit, Medical Oncology Department, Faculty of Medicine, University of L'Aquila. L'Aquila, Italy

3. Sport Science Unit, Faculty of Movement and Sport Sciences, University of L'Aquila. L'Aquila, Italy

4. Department of Experimental Medicine, University of L'Aquila, L'Aquila, Italy

Correspondence to: Marco Valenti MD, Biostatistica ed Epidemiologia, Facoltà Scienze Motorie, Dipartimento Medicina Interna e Sanità Pubblica, Via Cardinale Mazzarino 1, 67100 L'AQUILA, ITALY. Fax 00390862 432929; E-mail marco.valenti@cc.univaq.it.

Received: 2007.12.06; Accepted: 2008.01.08; Published: 2008.01.15

An important goal for cancer patients is to improve the quality of life (QOL) by maximising functions affected by the disease and its therapy. Preliminary research suggests that exercise may be an effective intervention for enhancing QOL in cancer survivors. Research has provided preliminary evidence for the safety, feasibility, and efficacy of exercise training in breast cancer survivors. The aim of this study was to assess the association between physical exercise and quality of life in a population of female breast cancer survivors, followed up from diagnosis to the off-treatment time period, and investigated about their exercise habits in pre-diagnosis.

A total of 212 female breast cancer survivors consecutively registered from January 2002 to December 2006 at a Supportive Care Unit in an Italian Oncology Department were enrolled. Exercise behaviour was assessed by the Leisure Score Index (LSI) of the Godin Leisure-Time Exercise Questionnaire. Patients were asked to report their average weekly exercise for three cancer-related time periods, i.e. pre-diagnosis, during active treatment and off-treatment. Quality of life was assessed by the Italian version of the WHOQOL-BREF standardised instrument. Statistical analysis indicated significant differences across the cancer-relevant time-periods for all exercise behaviour outcomes: the exercise behaviour was significantly lower during both on- and off- treatment than during prediagnosis; exercise during active treatment was significantly lower than during off-treatment. QOL strongly decreases during active treatment. Significant correlations were found between total exercise on- and off-treatment and all QOL indicators. Strenuous exercise is strongly correlated with QOL. Absent/mild exercise seems to be inversely correlated with a positive perception of disease severity and with quality of life on all axes. Need clearly results for inclusion of physical activity programs in comprehensive, complementary treatment regimes for breast cancer patients in Italian oncology departments.

Key words: Physical exercise, quality of life, breast cancer survivors, Italian Oncology

\section{INTRODUCTION}

Physical activity has many and varied effects on the human body. The physiological effects of physical activity and exercise in persons with cancer have been largely unstudied. In recent years, increased attention has focused on physical exercise as a rehabilitative intervention for cancer survivors during [1-5] and following [6-7] the cessation of cancer therapy.

The rationale posed for physical activity interventions following cancer diagnosis relates to minimizing biological processes associated with cancer promotion [8], enhancing behavioural changes linked with minimising lifestyle risk factors for recurrence of cancer [9], and improving psychosocial factors during and after cancer treatments [10].

Cancer patients as a group are at risk for diseases and conditions related to lack of physical activity.
Literature has focused on the main physiologic outcomes of physical activity after cancer: in persons with cancer, exercise has been shown to improve fitness and physical functioning, reduce fatigue, and modestly decrease weight and body fat [11]. The effects of physical activity on prognosis, however, are unknown. Increased physical activity reduces risk for several common cancers, which is relevant to cancer survivors who are at increased risk for new primary cancers. Risks of increased physical activity in cancer patients and survivors have not been defined, but could be expected to include muscular-skeletal injuries, and a small increased risk in sudden death with vigorous exercise and serious accidents with some sports. The effect of physical activity on survival from cancer is unknown, but physical activity might improve prognosis through beneficial effect on cancer 
biomarkers and energy balance, as well as decreasing risk for cardiovascular disease, an important cause of death for many cancer survivors.

Overall, literature provides promising but preliminary evidence that exercise may be a beneficial supportive therapy for cancer patients [12-13]. It is important to recognize that disease- and treatment-related responses vary between tumor site due to the pathophysiology of the neoplasm, specific treatment side effects, and demographic profile of patients. Thus generalizations between cancer sites may not be prudent [14].

There is still a lack of knowledge about the optimal types, duration, frequency, and timing of exercise [15-16]. The aerobic component has been emphasised in physical exercise programmes and health promotion in general, to the detriment of other types of exercise, such as strength training [17].

An important goal for cancer patients is to improve the quality of life (QOL) by maximising functions affected by the disease and its therapy $[6,10]$. Therefore, it is important to understand the effect of different exercise regimes on cancer patients at different stages of disease and treatment.

Health-related QOL, an outcome measure increasing in popularity in the health sciences including exercise science [18-21], has evolved to include aspects of life that affect perceived physical or mental health, and it is a fundamental measure used to understand a population's health status [22].

There is a growing interest in the possible role of exercise in enhancing QOL, reducing recurrence and other diseases, and extending survival in cancer survivors. Preliminary research suggests that exercise may be an effective intervention for enhancing QOL in cancer survivors and that this effect may be beyond that provided by group psychotherapy.

The studies are few in number, however, and of limited quality. Further research using rigorous methodology is needed to definitively answer questions concerning the role of exercise in enhancing QOL in cancer survivors both during and after treatment [12].

Breast cancer is a prevalent disease [23] that requires intense and prolonged treatments [24]. Cardiopulmonary capacity may be compromised in breast cancer survivors because of the pathology of the disease, therapeutic regimens, and weight gain and inactivity secondary to treatment. The reduction in cardiopulmonary capacity may lead to reductions in quality of life [25, 26]. Research has provided preliminary evidence for the safety, feasibility, and efficacy of exercise training in breast cancer survivors $[6,27]$. Few investigations, however, have been designed as follow up observational studies or randomised controlled trials. Overall, little is known about the impact of exercise training on QOL in breast cancer survivors.

The aim of this study was to assess the association between physical exercise and quality of life in a population of female breast cancer survivors, followed up from diagnosis to the off-treatment time period, and investigated about their exercise habits in pre-diagnosis.

\section{PATIENTS AND METHODS}

\section{Setting}

This study was conducted at the Supportive Care Unit of the University Hospital Oncology Department of L'Aquila, in central Italy. The target population of participants consisted of all female breast cancer survivors, who had received a stage I-II diagnosis, residing in the L'Aquila Health District. Hospital's Ethical Committee approval to the protocol was obtained.

Eligible participants were identified through the local hospital-based cancer registry. Twenty-one patients with cognitive impairment $(n=3)$ or chronic morbidities $(n=18)$ were excluded. Each eligible participant was sent a questionnaire package that contained detailed instructions, a consent form, and a questionnaire. Survey methods known to increase response rates were used. Eventually, a total of 212 female breast cancer survivors consecutively registered from January 2002 to December 2006 were enrolled. 23 out of $212(10.8 \%)$ withdrew from the follow up.

Demographic information was obtained by self-report measure. Medical information was obtained by hospital records.

\section{Quality of life}

Overall QOL was assessed by the Italian version of the World Health Organisation Quality of Life BREF assessment instrument (WHOQOL-BREF) [28-29]. The WHOQOL-BREF instrument provides an assessment of QOL in four areas (physical, psychological, social relationships, environmental): areas are structured in relevant sections. Each section contributes to the calculation of the overall area score, ranging from 0 to 100.

The assessment of QOL was performed at three months from diagnosis (during active treatment) and at three months after the end of active treatment (off-treatment period).

\section{Exercise behaviour}

Exercise behaviour was assessed by the Leisure Score Index (LSI) of the Godin Leisure-Time Exercise 
Questionnaire [30-31]. The LSI assesses the average frequency of mild (minimal effort), moderate (not exhausting) and strenuous intensity exercise during free time in a week. Patients were asked to report their average weekly exercise for three cancer-related time periods, i.e. pre-diagnosis, during active treatment and off-treatment. This way of administrating LSI has been recently validated in the context of cancer patients [14]. Patients were informed by the oncologists about facilities to carry out physical exercise in a specialised context offered by the L'Aquila Faculty of Sport Science: $35 \%$ of patients participated in custom-made exercise protocols.

\section{Statistical analysis}

Potential differences between basic characteristics and exercise behaviour and QOL were assessed by multivariate analysis of variance. Differences in exercise behaviour among the cancer-relevant time periods were analysed by repeated measure analysis of variance. Standard pairwise comparisons were performed to assess differences between groups. The association between total exercise during active treatment and off-treatment and QOL was assessed by correlation coefficients.

\section{RESULTS}

The mean age of participants was 55.1 years (standard deviation 6.2 years, range $42-65$ ); $90.0 \%$ were married, $41.0 \%$ had completed high school and $11.0 \%$ university, $51.2 \%$ were currently employed $(48.8 \%$ housekeepers/unemployed).

The mean follow up time since diagnosis was 2.6 years (sd 0.9 years). $100 \%$ of patients had completed surgery (partial mastectomy), 23\% had received radiation and $74 \%$ had received chemotherapy.

Multivariate analysis of variance indicated no association between baseline patients' characteristics and both exercise behaviour and QOL (data not shown).

Table 1 describes exercise behaviour across cancer-related time periods. The overall repeated-measures MANOVA for all exercise behaviour outcomes across the cancer relevant time periods was significant at $\mathrm{p}<0.001 \quad(\mathrm{~F}=5.16)$; also the ANOVAs indicated significant differences across the cancer-relevant time-periods for all exercise behaviour outcomes. Pairwise comparisons indicated that exercise behaviour was significantly lower during both on- and off- treatment than during prediagnosis; exercise during active treatment was significantly lower than during off-treatment.

Table 2 shows mean values of QOL indicators across the two clinical cancer-relevant time-periods. As largely known, QOL strongly decreases during active treatment.

Table 3 displays correlations between exercise during active treatment and QOL. Analyses show significant correlations between total exercise and all QOL indicators. Strenuous exercise is strongly correlated with QOL. Interestingly, mild exercise seems to be inversely correlated with a positive perception of disease severity.

Table 4 displays correlations between exercise during off-treatment and QOL. Results are similar than on treatment. It is confirmed that absent/mild exercise correlates inversely with quality of life on all axes.

Table 1. Exercise behaviour across cancer-related time periods.

\begin{tabular}{|c|c|c|}
\hline Weekly exercise prediagnosis (min) & mean & sd \\
\hline Total & $213.7{ }^{*} \#$ & 280.9 \\
\hline Strenuous & 56.7 & 129.0 \\
\hline Moderate & 120.4 & 180.1 \\
\hline Mild & 136.6 & 199.2 \\
\hline Weekly exercise during treatment (min) & & \\
\hline Total & $87.0 \quad$ * § & 170.2 \\
\hline Strenuous & 6.7 & 88.1 \\
\hline Moderate & 23.4 & 126.5 \\
\hline Mild & 56.9 & 179.0 \\
\hline Weekly exercise during off-treatment (min) & \multicolumn{2}{|c|}{} \\
\hline Total & 189.5 § \# & 263.5 \\
\hline Strenuous & 31.6 & 108.9 \\
\hline Moderate & 67.7 & 138.2 \\
\hline Mild & 90.2 & 199.0 \\
\hline
\end{tabular}

Overall repeated-measures MANOVA (exercise behaviour outcomes across time periods) $\mathrm{p}<0.001$ ( $\mathrm{F}=5.16)$;

Pairwise comparisons * $\mathrm{p}<0.001 \S \mathrm{p}<0.001$ \# $\mathrm{p}<0.05$

Table 2. QOL across clinical follow up

\begin{tabular}{|c|c|c|}
\hline QOL during active treatment & & \\
\hline WHOQOL-BREF score & mean & sd \\
\hline Physical area & 53.45 & 16.02 \\
\hline Psychological area & 66.56 & 14.33 \\
\hline Social relationships area & 59.87 & 15.59 \\
\hline Environmental area & 65.61 & 17.12 \\
\hline QOL during off-treatment & & \\
\hline WHOQOL-BREF score & mean & sd \\
\hline Physical area & 59.83 & 16.34 \\
\hline Psychological area & 70.12 & 15.99 \\
\hline Social relationships area & 62.63 & 16.29 \\
\hline Environmental area & 66.84 & 17.05 \\
\hline
\end{tabular}

Table 3. Correlations between exercise and QOL during active treatment

\begin{tabular}{|c|c|c|c|c|}
\hline $\begin{array}{c}\text { Exercise } \\
\text { behaviour }\end{array}$ & $\begin{array}{c}\text { Physical } \\
\text { area }\end{array}$ & $\begin{array}{c}\text { Psychological } \\
\text { area }\end{array}$ & $\begin{array}{c}\text { Social } \\
\text { relationships } \\
\text { area }\end{array}$ & $\begin{array}{c}\text { Environmental } \\
\text { area }\end{array}$ \\
\hline Total & $0.57^{* *}$ & $0.46^{* *}$ & $0.52^{* *}$ & $0.55^{* *}$ \\
\hline Strenuous & $0.41^{* *}$ & $0.32^{*}$ & $0.44^{* *}$ & $0.45^{* *}$ \\
\hline Moderate & $0.28^{*}$ & 0.20 & 0.16 & $0.30^{*}$ \\
\hline Mild & -0.19 & $-0.27^{*}$ & -0.21 & $0.28^{*}$ \\
\hline
\end{tabular}


Table 4. Correlations between exercise and QOL during off-treatment

\begin{tabular}{|c|c|c|c|c|}
\hline $\begin{array}{c}\text { Exercise } \\
\text { behaviour }\end{array}$ & $\begin{array}{c}\text { Physical } \\
\text { area }\end{array}$ & $\begin{array}{c}\text { Psychological } \\
\text { area }\end{array}$ & $\begin{array}{c}\text { Social } \\
\text { relationships } \\
\text { area }\end{array}$ & $\begin{array}{c}\text { Environmental } \\
\text { area }\end{array}$ \\
\hline Total & $0.60^{* *}$ & $0.51^{* *}$ & $0.51^{* *}$ & $0.63^{* *}$ \\
\hline Strenuous & $0.44^{* *}$ & $0.33^{*}$ & $0.45^{* *}$ & $0.51^{* *}$ \\
\hline Moderate & $0.32^{*}$ & 0.16 & 0.17 & $0.37^{*}$ \\
\hline Mild & $-0.29^{*}$ & $-0.34^{*}$ & $-0.27^{*}$ & $0.35^{*}$ \\
\hline${ }^{*} \mathrm{p}<0.05$ & ${ }^{* *} \mathrm{p}<0.01$ & & & \\
\hline
\end{tabular}

\section{DISCUSSION}

Research into the possible role of exercise in cancer survivors is of relatively recent vintage compared with research examining exercise and cancer prevention. Evidence for physical exercise as a supportive therapy for breast cancer survivors is growing, also on the basis of observational studies based on self-reported measures of leisure-time physical activity. Literature offers preliminary evidence of the positive effects of exercise on QOL for both on-treatment and off-treatment follow-up periods in breast cancer: such evidence is strongly confirmed by our work.

Reservations could be presented about the use of self-reported questionnaires. The self-reported questionnaire is the most common instrument in physical activity records, because it is easy-to-use, short to perform, inexpensive, and suitable for epidemiological purposes. An important issue of self reported questionnaires is the respondent's ability to make an accurate assessment of physical activity. Indeed, the LSI is an established validated instrument for general and clinical setting, and ensures accurate assessment of physical activity on a quantitative basis. Recently, it has been demonstrated that social desirability and social approval may influence self-reported physical activity on some survey instruments [32].

A side methodological issue is the conduct of intervention studies (experimental or quasi-experimental studies). Apart from the relatively low number of controlled trials on physical exercise as a component of treatment, some reviews have focused on important methodological problems concerning patient recruitment and compliance, and the ability to generalise from the results [33,34]. Patient withdrawal during intervention is a general problem in experimental research, and of special relevance in physical exercise interventions. In healthy populations, approximately $50 \%$ of those who start an exercise programme drop out during the first six months [35]. A large drop-out rate reduces the sample's representativity of the larger population, the strength of the findings and the ability to generalise from the results. Even if our study was observational, we ensured the participants to take advantage of supervised physical activity in the context of follow up: $35 \%$ of patients exploited the opportunity, so that questionnaire recalls of physical activity intensity and duration resulted easier. In our study only $10.7 \%$ of participants withdrew from the study: this surprisingly positive finding may be explained in terms of perceived quality of care related to the offering of custom-made specialised exercise protocols.

Short duration interventions are a general problem in exercise programmes. Some interventions are too short to produce any effects. However, this depends on how the primary endpoint of the intervention is defined. For endpoints that include subjective outcomes such as QOL measures, the instrument's dimension(s) or selected endpoint(s) should be identified before subjects are recruited.

Relatively few studies have examined the feasibility and potential health benefits of supervised, structured exercise programs for sedentary women with primary breast cancer. Results from Kolden et al. [36] show that participants in group exercise training (not self-administered) experience significant health benefits over the course of the intervention in multiple dimensions of fitness/vigor (aerobic capacity, strength, flexibility) as well as QOL (increased positive affect, decreased distress, enhanced well-being, and improved functioning).

Overall, we believe in agreement with Courneya [12] that there is sufficient evidence for breast cancer patients to warrant second generations studies focusing on more specific questions such as optimal timing, type, frequency and duration of physical exercise. Studies are also needed to examine mechanisms of change for QOL and to compare and integrate exercise with other currently accepted QOL interventions.

To date, this is the first study on physical exercise and breast cancer conducted in an Italian oncology setting. Taking together the promising findings from our study and the literature, need clearly results for inclusion of physical activity programs in comprehensive, complementary treatment regimes for breast cancer patients in Italian oncology departments.

\section{CONFLICT OF INTEREST}

The authors have declared that no conflict of interest exists.

\section{REFERENCES}

1. Dimeo FC, Tilmann MH, Bertz H, Kanz L, Mertelsmann R, Keul $\mathrm{J}$. Aerobic exercise in the rehabilitation of cancer patients after high dose chemotherapy and autologous peripheral stem cell 
transplantation. Cancer. 1997;79:1717-22.

2. Dimeo FC, Stieglitz RD, Novelli-Fischer U, Fetscher S, Keul J. Effects of physical activity on the fatigue and psychologic status of cancer patients during chemotherapy. Cancer. 1999;85:2273-7.

3. Mock V, Dow KH, Meares CJ, Grimm PM, Dienemann JA, Haisfield-Wolfe ME, Quitasol W, Mitchell S, Chakravarthy A, Gage I. Effects of exercise on fatigue, physical functioning, and emotional distress during radiation therapy for breast cancer. Oncol Nurs Forum. 1997;24:991-1000.

4. Mock V, Pickett M, Ropka ME, Muscari Lin E, Stewart KJ, Rhodes VA, McDaniel R, Grimm PM, Krumm S, McCorkle R. Fatigue and quality of life outcomes of exercise during cancer treatment. Cancer Pract. 2001;9:119-27.

5. Segal R, Evans W, Johnson D, Smith J, Colletta S, Gayton J, Woodard S, Wells G, Reid R. Structured exercise improves physical functioning in women with stages I and II breast cancer: results of a randomized controlled trial. J Clin Oncol. 2001;19:657-65.

6. Courneya KS, Mackey JR, Bell GJ, Jones LW, Field CJ, Fairey AS. Randomized controlled trial of exercise training in postmenopausal breast cancer survivors: cardiopulmonary and quality of life outcomes. J Clin Oncol. 2003;21:1660-8.

7. Segal RJ, Reid RD, Courneya KS, Malone SC, Parliament MB, Scott CG, Venner PM, Quinney HA, Jones LW, D'Angelo ME, Wells GA. Resistance exercise in men receiving androgen deprivation therapy for prostate cancer. J Clin Oncol. 2003;21:1653-9.

8. Nieman DC, Cook VD, Henson DA, Suttles J, Rejeski WJ, Ribisl PM, Fagoaga OR, Nehlsen-Cannarella SL. Moderate exercise training and natural killer cell cytotoxic activity in breast cancer patients. Int J Sports Med. 1995;16:334-7.

9. Stoll BA. Diet and exercise regimens to improve breast carcinoma prognosis. Cancer. 1996;78:2465-70.

10. Courneya KS. Exercise interventions during cancer treatment: biopsychosocial outcomes. Exerc Sport Sci Rev. 2001;29:60-4.

11. McTiernan A. Physical activity after cancer: physiologic outcomes. Cancer Invest. 2004;22:68-81.

12. Courneya KS. Exercise in cancer survivors: an overview of research. Med Sci Sports Exerc. 2003;35:1846-52.

13. Oldervoll LM, Kaasa S, Hjermstad MJ, Lund JA, Loge JH. Physical exercise results in the improved subjective well-being of a few or is effective rehabilitation for all cancer patients? Eur J Cancer. 2004;40:951-62.

14. Jones LW, Courneya KS, Vallance JK, Ladha AB, Mant MJ, Belch AR, Stewart DA, Reiman T. Association between exercise and quality of life in multiple myeloma cancer survivors. Support Care Cancer. 2004;12:780-8.

15. Courneya KS, Friedenreich CM. Physical exercise and quality of life following cancer diagnosis: a literature review. Ann Behav Med. 1999;21:171-9.

16. Brown DW, Brown DR, Heath GW, Balluz L, Giles WH, Ford ES, Mokdad AH. Associations between Physical Activity Dose and Health-Related Quality of Life. Med Sci Sports Exerc. 2004;36:890-6

17. Blair SN, Kampert JB, Kohl HW 3rd, Barlow CE, Macera CA, Paffenbarger RS Jr, Gibbons LW. Influences of cardiorespiratory fitness and other precursors on cardiovascular disease and all-cause mortality in men and women. JAMA. 1996;276:205-10.

18. Rejeski WJ, Brawley IR, Schumaker SA. Physical activity and health-related quality of life. In: Holloszy JO, ed. Exercise and Sport Sciences Reviews, Vol. 24. Baltimore: Williams \& Wilkins; 1996: 71-108.

19. Spirduso WW, Cronin DL. Exercise dose-response effects on quality of life and independent living in older adults. Med Sci Sports Exerc. 2001;33(S):598 -608.

20. Trine MR. Physical activity and quality of life. In: Rippe JM, ed. Lifestyle Medicine. Malden: Blackwell Science Inc; 1999:989-97.
21. Twelves CJ, Miles DW, Hall A. Quality of life in women with advanced breast cancer treated with docetaxel. Clin Breast Cancer. 2004;5:216-22

22. US Centers for Disease Control and Prevention. Measuring Healthy Days: Population Assessment of Health-Related Quality of Life. Atlanta, GA: USCenters for Disease Control and Prevention. 2000:1-44.

23. American Cancer Society. Cancer Facts and Figures. Atlanta, GA: American Cancer Society; 2001

24. Hortobagyi GH. Treatment of breast cancer. N Engl J Med. 1998;339:974-84

25. Shapiro CL, Recht A. Side effects of adjuvant treatment of breast cancer. N Engl J Med. 2001 344:1997-2008

26. Zabora J, Brintzenhofeszoc K, Curbow B, et al. The prevalence of psychological distress by cancer site. Psychooncology. 2001;10:19-28

27. Courneya KS, Mackey JR, McKenzie DC. Exercise after breast cancer: Research evidence and clinical guidelines. Physician Sportsmed. 2002,30:33-42

28. The WHOQOL Group. Development of the WHOQOL-BREF Quality of Life Assessment. Psychological Medicine. 1998;28:551-8

29. De Girolamo G, Rucci P, Scocco P, Becchi A, Coppa F, D'Addario A, Darú E, De Leo D, Galassi L, Mangelli L, Marson C, Neri G, Soldani L. Quality of life assessment: validation of the Italian version of the WHOQOL-Bref. Epidemiol Psichiatr Soc. 2000;9:45-55.

30. Godin G, Shephard RJ. A simple method to assess exercise behaviour in the community. Can J Appl Sport Sci. 1985;10:141-6.

31. Godin G, Jobin J, Bouillon J. Assessment of leisure time exercise behaviour by self-report: a concurrent validity study. Can J Public Health. 1986;77:359-62

32. Adams SA, Matthews CE, Ebbeling CB, Moore CG, Cunningham JE, Fulton J, Herbert JR. The effect of social desirability and social approval on self-reports of physical activity. Am J Epidemiol. 2005;161:389-98

33. Irwin ML, McTiernan A, Bernstein L, Gilliland FD, Baumgartner R, Baumgartner K, Ballard-Barbash R. Physical activity levels among breast cancer survivors. Med Sci Sports Exerc. 2004;36:1484-91.

34. Irwin ML, Ainsworth BE. Physical activity interventions following cancer diagnosis: methodologic challenges to delivery and assessment. Cancer Invest. 2004;22:30-50.

35. Dishman DK. Determinants of participation in physical activity. In: Bouchard C, Shepard RJ, Stephens T, Sutton JR, McPherson BD, eds. Exercise, Fitness and Health: A Consensus of Current Knowledge. Champaign IL: Human Kinetics; 1990:75-101

36. Kolden GG, Strauman TJ, Ward A, Kuta J, Woods TE, Schneider KL, Heerey E, Sanborn L, Burt C, Millbrandt L, Kalin NH, Stewart JA, Mullen B. A pilot study of group exercise training (GET) for women with primary breast cancer: feasibility and health benefits. Psychooncology. 2002;11:447-56. 